Service social

\title{
Participation ou retrait de la personne âgée?
}

\section{Jacques Laforest}

Volume 34, numéro 1, 1985

Personnes âgées, milieux de vie et pratiques sociales

URI : https://id.erudit.org/iderudit/706246ar

DOI : https://doi.org/10.7202/706246ar

Aller au sommaire du numéro

Éditeur(s)

École de service social de l'Université Laval

ISSN

1708-1734 (numérique)

Découvrir la revue

Citer cet article

Laforest, J. (1985). Participation ou retrait de la personne âgée? Service social, 34(1), 4-10. https://doi.org/10.7202/706246ar d'utilisation que vous pouvez consulter en ligne.

https://apropos.erudit.org/fr/usagers/politique-dutilisation/ 


\section{Participation ou retrait de la personne âgée?}

\section{Jacques Laforest}

En termes de services sociaux et d'aide psychosociale, les besoins des personnes âgées sont considérables, et l'on ne peut qu'en prévoir l'augmentation constante. Cette situation provoque une importante mobilisation des ressources de la société en leur faveur, mais cela ne va pas sans ambiguïté.

\section{Conditions d'une pratique nouvelle}

La situation est telle que les travailleurs sociaux se trouvent devant un véritable défi à relever : découvrir la voie d'une pratique nouvelle. En gérontologie, les praticiens, les planificateurs et les chercheurs s'attaquent à des problèmes ponctuels à l'intérieur d'une problématique plus profonde et plus globale dont ils sont prisonniers : nous vivons dans un contexte où il n'y a pas de place pour les personnes âgées.

Pour être adaptée à leurs vrais besoins, la pratique du service social se doit d'aller à contre-courant, c'est-à-dire de refuser de se laisser emprisonner dans l'attitude générale d'une société qui se révèle incapable de définir une place significative pour la génération de ses aînés. Au cours des cinquante dernières années, un changement profond des institutions et des valeurs s'est produit. C'est devenu un cliché de dire cela ; tout le monde admet l'existence et la profondeur de ce phénomène de mutation socioculturelle comme une évidence. Mais nous ne discernons pas aussi clairement toutes ses implications et nous avons encore moins acquis le contrôle de ses conséquences.

Pour les personnes âgées, on commence à s'éveiller à une brutale réalité : l'ancienne société n'existe plus, où l'expérience de la vieillesse était valorisée, où le processus du vieillissement se traduisait par une adaptation correspondante de la participation sociale dans le contexte de la famille étendue et de l'entreprise familiale ou corporative, au sein 
de groupes sociaux aux relations non spécialisées et suivant un déroulement du temps ponctué par le rythme des fêtes et des saisons. Mais quelle est leur nouvelle place si elle ne peut plus être celle d'autrefois? Celle qu'elles avaient autrefois est disparue sans avoir été remplacée: plus précisément, les aînés expérimentent qu'ils n'ont pas de place dans leur propre société.

Considérons les trois principaux domaines d'activité du service social, comme discipline et comme profession : les politiques sociales, la recherche et la pratique professionnelle. Les premières se sont développées au cours des dernières années, mais il reste encore beaucoup à faire. Les coûts des services sociaux vont sans doute encore augmenter; mais tant que ces investissements seront en réalité des compensations pour une situation réelle de dévalorisation et d'exclusion, leur efficacité potentielle pour le bien-être des personnes visées sera neutralisée. La recherche aussi se développe rapidement : évaluation de programmes, description de problèmes, sondage d'opinions et d'attitudes, accumulation de statistiques. Les résultats de cet effort de développement des connaissances resteront assez décevants tant qu'on cherchera à connaître des moyens plus efficaces pour rendre plus épanouissante et moins coûteuse socialement une situation de dévaluation et d'exclusion. Quant à la pratique même des travailleurs sociaux, elle est pour le moins frustrante car : a) il faut adapter aux besoins de cette clientèle spécifique des services en quantité toujours insuffisante; b) il faut réclamer des ressources plus grandes et surtout plus adaptées, malgré les réticences de la société qui dévalorise la situation de dépendance où elle les confine ; c) il faut aussi, à supposer qu'on nous en laisse le temps, les aider à solutionner les problèmes psychosociaux les plus aigus et les plus urgents causés par leur vécu sans que leur rôle n'ait été défini.

Les trois domaines dont nous venons de parler ont une valeur intrinsèque indéniable. Mais, pour être vraiment efficaces, ils doivent répondre à la condition fondamentale d'une pratique transformée, c'est-à-dire être conditionnés et influencés par un même objectif ultime : aider à la définition d'une nouvelle place pour les personnes âgées dans une société nouvelle.

\section{Participation sociale adaptée}

Aujourd'hui, le statut social courant d'un aîné est celui de "retraité" qui est, par définition, négatif puisqu'il ne signifie rien d'autre que "le retrait de...". Le concept de "place dans la société», vu comme positif 
et dynamique, suggère l'idée d'appartenance à un groupe, de consensus avec lui, de complicité affective, émotionnelle, sociale et professionnelle.

Le vieillissement est une œuvre de la nature tandis que l'association vieillesse-retraite ne l'est pas. Quand on dit retraite, on pense tout naturellement au départ du marché du travail; plus que cela, c'est l'absence de participation sociale, dans toutes les dimensions de la vie. Aussi est-ce un processus progressif et graduel, qui commence ordinairement lorsqu'on quitte son travail, souvent même avant, et qui est vécu comme une expérience cumulative d'exclusion.

Les forces diminuent avec l'âge, entraînant des circonstances différentes à affronter et des besoins nouveaux à satisfaire. Cette situation n'est pas vécue comme une aspiration à délaisser la participation sociale mais est ressentie, plutôt, comme un besoin d'adapter celle-ci. Le défi à relever, en gérontologie, est donc la définition de la place des personnes âgées en termes de participation adaptée. II ne s'agit donc pas seulement ici des centres d'accueil ou autres ressources d'hébergement (cela c'est le lieu physique, le parc de stationnement), mais de leur rôle significatif - pas nécessairement productif selon les critères de l'éthique fonctionnaliste. On parle de ce que les personnes âgées sont dans et pour cette société. Ainsi comprise, leur place serait donc la source de la satisfaction de leurs besoins d'appartenance, de valorisation et de motivation. Cela est vrai pour tous, non seulement pour les aînés d'ailleurs.

Adaptation ou retrait de la participation sociale? Nos pratiques sociales en gérontologie s'inspirent de la perspective du retrait et prévoient des recours matériels pour rendre ce désengagement le plus confortable possible: ressources d'hébergement, soutien du revenu, soins de santé. II ne faut pas s'étonner que ces pratiques collectives soient toujours insuffisantes puisque, bien que nécessaires et valables en soi, elles sont prisonnières d'une philosophie du vieillissement qui neutralise leur efficacité réelle. En effet, même quand on y investit plus de ressources, nos pratiques sociales collectives dans ce domaine restent des compensations pour une situation de dévalorisation et d'exclusion.

\section{Symptômes d'une attitude collective}

Il existe chez nous deux documents officiels que l'on peut considérer comme les témoins les plus récents et les plus révélateurs des perspectives fondamentales qui sous-tendent nos politiques et programmes sociaux pour les personnes âgées. Le premier émane du 
Gouvernement fédéral et a été publié en 1979 par un comité spécial du Sénat: Retraite sans douleur; le second a été rendu public par le Gouvernement du Québec, en 1980: Pour mieux vieillir au Québec. Sur le plan de la consultation publique, les deux paliers de gouvernement ont suivi un cheminement inverse : le document émanant du niveau provincial a d'abord été préparé par le Ministère des affaires sociales, et ensuite soumis à la consultation; quant à celui du Fédéral, le comité spécial du Sénat a d'abord commencé par tenir des audiences publiques à travers tout le pays avant que le rapport proprement dit ne soit rédigé et publié. Pour ce qui est du contenu, Pour mieux vieillir au Québec est un document très succinct présenté comme un projet global de politique du troisième âge, y compris la question particulière de l'âge de la retraite. Par contre, même s'il est beaucoup plus élaboré, Retraite sans douleur se propose de circonscrire ses interrogations de départ à cette même question ; mais, comme on s'en doute bien, on ne peut proposer une politique de l'âge de la retraite séparément d'une politique plus globale de la retraite comme telle.

Donc, malgré les apparences, les deux documents proposent la même chose : une politique d'ensemble de la retraite, abordée par le biais du moment de transition vers celle-ci. Il ne faut pas manquer de souligner, au passage, cette perspective commune aux deux documents. Pour définir une politique de la vieillesse, on définit une politique de la retraite : sans doute identifie-t-on les deux concepts. Cette perspective révèle du même coup la place, ou tout au moins le statut qu'on assigne aux personnes âgées dans la société : celui de "retraitées".

En plus de quelques orientations pour la pratique, les deux documents proposent une même politique, soit de supprimer l'âge obligatoire de la retraite, et ce, à peu près pour les mêmes raisons : ils fondent leur recommandation, d'abord, sur un principe : celui du libre choix, à l'exclusion de la discrimination fondée sur l'âge, et sur celui de la continuité des conditions d'existence matérielles et sociales. Ensuite, ils apportent une justification empirique: l'âge obligatoire de la retraite à 65 ans entraîne des maux sociaux pour cette catégorie de la population, entre autres une chute soudaine et importante de revenu, jointe à une dévalorisation sociale. Quant aux orientations pour la pratique, ils proposent plusieurs modalités pour introduire leur nouvelle politique. Toutes ces formules ont en commun de viser le même objectif : rendre progressive la transition à la retraite définitive en facilitant ce passage du point de vue financier.

Au Québec, suite à une promesse électorale, l'âge obligatoire de la retraite est supprimé, du moins en principe, par l'entrée en vigueur, le $1^{\text {er }}$ avril 1982, de la Loi 15 . Ce ne sont toutefois pas les textes légaux 
eux-mêmes qui ont un impact réel sur le vécu des personnes âgées, mais plutôt la philosophie du vieillissement dont ils émanent. Or, la Loi 15 reflète la même philosophie que Pour mieux vieillir au Québec et Retraite sans douleur. Le statut de retraité accolé aux personnes âgées, dans notre société, demeure donc inchangé; on cherche seulement à l'améliorer en favorisant une transition progressive, dans les meilleures conditions monétaires possibles. Au fond, la vraie question qui se pose ici est celle du libre choix de l'âge de la retraite. Or, en fonction de cela, il faut au moins deux conditions : avoir les moyens de choisir, et, aussi, avoir deux alternatives.

Pour ce qui est des moyens préalables à cette liberté, on ne voit pas, dans les documents cités plus haut, comment le simple fait de supprimer l'obligation de la retraite à 65 ans va donner du travail aux chômeurs de 50 ans et plus, pour qui elle devient obligatoire à un âge anticipé. Il y a aussi les salariés âgés qui ont un emploi mais pas les moyens de se retirer; il ne faudrait pas parler de liberté, si on remplace l'obligation de prendre sa retraite par celle de ne pas la prendre. La loi pourrait introduire la liberté de choix comme principe, mais son exercice pourrait fort bien être limité par différents facteurs tels l'affaiblissement physiologique, la pauvreté, ou encore, simplement l'absence de possibilités de travail.

Mais, à supposer que tous auraient les "moyens" de choisir, que feraient-ils? En raison des pertes inhérentes au processus de vieillissement, chacun sent, tôt ou tard, une pression grandissante, de l'intérieur et de l'extérieur, à modifier le mode de participation sociale qui a été le sien jusque là. Comme en témoignent les deux documents que nous venons d'analyser, le choix que notre société leur offre n'est pas entre deux formes égales, mais entre la participation et le retrait.

Le retrait du marché du travail est une étape importante dans ce processus de rupture de la participation sociale. Mais ce n'est pas la seule. Le processus, une fois initié, se continue inexorablement jusqu'à l'étape absolue : la mort. La plupart du temps, la personne vieillissante est entrée dans un stade de dévalorisation plusieurs années avant l'éloignement du marché du travail, à cause de la dévaluation de l'expérience. De plus, dans notre contexte économique, elle est perçue comme tenant un emploi à la place des jeunes; et une fois retirée du marché du travail, elle représente encore un handicap en regard des valeurs économiques dominantes, à cause du poids démographique de la vieillesse.

La Loi 15 et les deux documents déjà cités manifestent bien l'attitude générale de notre société, sans une vraie place qui se définirait par un type quelconque mais réel de participation sociale. II 
existe bien d'autres manifestations de cette attitude. D'abord, la Loi 15 n'a pas été votée à l'unanimité, la plupart de ses opposants invoquant la nécessité de soustraire encore plus tôt du marché du travail les personnes âgées pour alléger le poids du chômage des jeunes. On ne proposait pas d'alternative en termes de participation adaptée; toujours l'exclusion pure et simple, consécutive à une dévalorisation. D'ailleurs, il faudrait voir comment s'appliquent, dans les faits, les dispositions de la Loi 15. Tous ces discours officiels concernant les "bienfaits" de la retraite anticipée sont pour le moins fort ambigus.

Par exemple, dans son édition du 14 avril 1983, Le Soleil de Québec rapportait : "la recommandation que la Conférence des recteurs et principaux d'universités a récemment faite au gouvernement, réclamant le décret d'un moratoire dans l'application de la Loi 15 et autorisant les universités à donner congé à leurs professeurs qui ont atteint l'âge normal de la retraite». Le journaliste rapportait ensuite une déclaration d'un porte-parole de cet organisme, à l'effet que "le maintien au travail des professeurs de plus de 65 ans est un obstacle majeur au développement de la recherche scientifique dans les universités... II s'en trouve environ 200 dans les universités de la province, et avec les crédits épargnés, il serait possible d'embaucher, à un salaire deux fois moindre, pas moins de 400 jeunes chercheurs, une source de dynamisme et de vitalité absolument nécessaire à la prise du tournant technologique».

Une telle prise de position n'a pas de quoi étonner. Elle reflète seulement avec une franchise plus brutale le statut assigné à la génération des aînés : une dévalorisation conduisant à l'exclusion. On ne saurait formuler d'une façon plus explicite un constat d'impuissance à définir la place et le rôle des personnes âgées dans notre contexte social.

\section{Élargir le concept de recherche}

Ceci est le cœur même de la problématique des personnes âgées comme champ de pratique du service social : au sortir d'un processus de profonde mutation, notre société découvre avec grand malaise qu'elle est incapable de définir une place pour la génération des aînés. Le statut de retraité est, au mieux, une compensation pour une situation réelle d'exclusion. Chez nous, on devient vieux parce qu'on est mis à la retraite et non l'inverse.

C'est dans ce contexte que le service social va devoir élaborer une pratique professionnelle inédite, et définir des orientations originales 
pour les politiques et programmes sociaux. Tout cela ne se fera pas sans un important effort de développement des connaissances. Le défi à relever est considérable: quel type de recherche pourrait être proportionné à une entreprise telle que la définition de la place des personnes âgées dans notre société en termes de participation sociale adaptée? Sans doute que les techniques habituelles de recherche en sciences sociales resteront fort utiles, indispensables. Mais, de forme quantitative et plus ou moins empruntées aux sciences physiques et mathématiques, elles ne suffiront pas à elles seules à identifier et interpréter des phénomènes qui se situent au plan de l'évolution des valeurs. À quoi bon prendre un virage technologique qui serait un substitut pour notre incapacité à prendre un virage humain?

Il faudra même aller plus loin. Le vieillissement, de par le dépouillement progressif qu'il impose, nous oblige à dépasser les phénomènes physiques, observables et mesurables, pour considérer la réalité métaphysique. Sous peine de ne pas répondre aux vrais besoins des personnes âgées parmi nous, notre effort de développement des connaissances doit s'appuyer sur des activités de recherche sur trois plans: physique ou phénoménologique, métaphysique, et ces deux niveaux en étant eux-mêmes inséparables, l'univers des valeurs. Ces trois types de recherche sont également nécessaires et valides en tant qu'activités proprement dites. Bien plus, en gérontologie, ils sont complémentaires de par leur nature même, et sont appelés à s'éclairer mutuellement. 